\title{
Fracture Nonunion Treated with Low-Intensity Pulsed Ultrasound and Monitored with Ultrasonography: A Feasibility Study
}

\author{
Peizhen Zhang, ${ }^{1}$ Pengdong $\mathrm{Li}^{2}{ }^{2}$ Shihai Liao, ${ }^{1}$ Xuan $\mathrm{Li}^{3}{ }^{3}$ Wufan Chen, ${ }^{1}$ Xiaoyun Wang $\mathbb{D},{ }^{2}$ \\ and Qing Wang $\mathbb{1}^{1}$ \\ ${ }^{1}$ School of Biomedical Engineering, Guangdong Provincial Key Laboratory of Medical Image Processing, Southern Medical University, \\ Guangzhou 510515, China \\ ${ }^{2}$ Guangdong Work Injury Rehabilitation Center, Guangzhou 510440, China \\ ${ }^{3}$ Nanfang Hospital, Southern Medical University, Guangzhou 510515, China
}

Correspondence should be addressed to Xiaoyun Wang; xiaoyunwang77@hotmail.com and Qing Wang; wq8740@smu.edu.cn

Received 10 September 2020; Revised 26 December 2020; Accepted 5 January 2021; Published 27 January 2021

Academic Editor: Yongjin Zhou

Copyright ( 92021 Peizhen Zhang et al. This is an open access article distributed under the Creative Commons Attribution License, which permits unrestricted use, distribution, and reproduction in any medium, provided the original work is properly cited.

The positive effect of low-intensity pulsed ultrasound (LIPUS) on bone fracture healing has been proved. However, during the period of LIPUS therapy, it is undetermined whether LIPUS promotes the formation of heterotopic ossification (HO), which usually occurs in muscle tissues after trauma such as bone fracture and spinal cord injury. Here, we used 6-week LIPUS therapy in a 42-year-old Chinese male patient with a fracture nonunion in combination with ultrasonography for monitoring fracture healing and HO formation. After the LIPUS therapy, the mineralized bone formation in the area of defect of the distal tibia was presented in an ultrasound image, which was consistent with the outcome of plain radiography showing callus formation and the blurred fracture line in the area exposed to LIPUS. In addition, ultrasound images revealed no evidence of HO development within soft tissues during the period of LIPUS therapy. This study suggests that ultrasonography is a potential tool to guarantee the performance of LIPUS therapy with monitoring HO formation. Easy to use, the integration of the handheld ultrasound scanner and the ultrasonic therapeutic apparatus is entirely dedicated to help orthopedists make high-quality care and diagnosis.

\section{Introduction}

With the increasing aging population and the rapid development of transportation and construction industry, trauma usually occurs and has become one of the major health problems. However, the prognosis of trauma such as bone fracture and spinal cord injury is sometimes poor. For example, the incomplete fracture healing along with a lack of radiological sign of bony continuity over three consecutive months after the initial rehabilitation treatment is diagnosed as nonunion $[1,2]$, which may not heal without any intervention [3]. The "gold standard" for the nonunion treatment is surgical intervention, usually including open surgical debridement of the nonunion site and application of internal or external fixation, in most cases with bone grafting [4]. The surgical intervention of nonunion is aimed at providing adequate mechanical stability and osteogenic environment to promote healing [5]. The success rate ranges from $68 \%$ to $96 \%$ varying by nonunion site and surgery type [4]. However, the technical difficulty and the risk of complications limit the use of the surgical intervention in nonunion $[2,5,6]$.

Previous studies demonstrated the effectiveness of fracture healing using low-intensity pulsed ultrasound (LIPUS). Kristiansen et al. reported a $38 \%$ reduction in the time of radiographic healing by the use of LIPUS to treat fractures [7]. In an animal model, LIPUS accelerated mature callus formation and the recovery of torsional stiffness and maximum torque [8]. Other studies investigated the mechanism of acceleration of fracture healing using LIPUS. Rawool et al. revealed that LIPUS might promote angiogenesis and increase blood supply in the tissues around the fracture [9]. It was also found that LIPUS was able to enhance adenylate cyclase activity, to transform growth factor- $\beta$ synthesis in osteoblasts [10], and to alter aggrecan gene expression to 


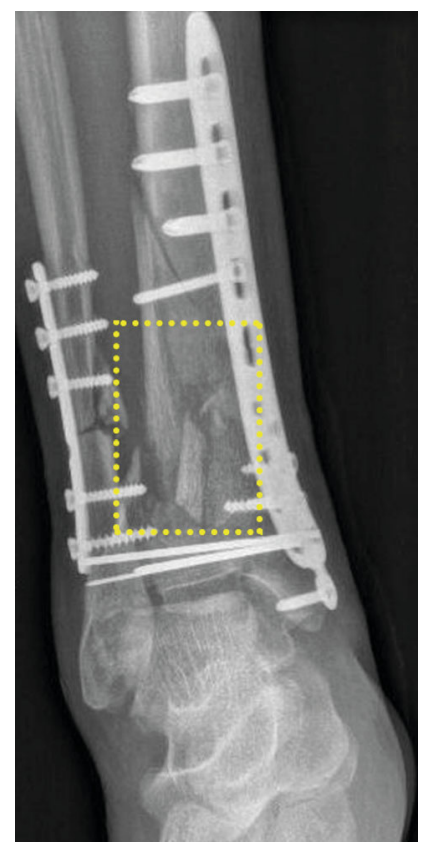

(a)

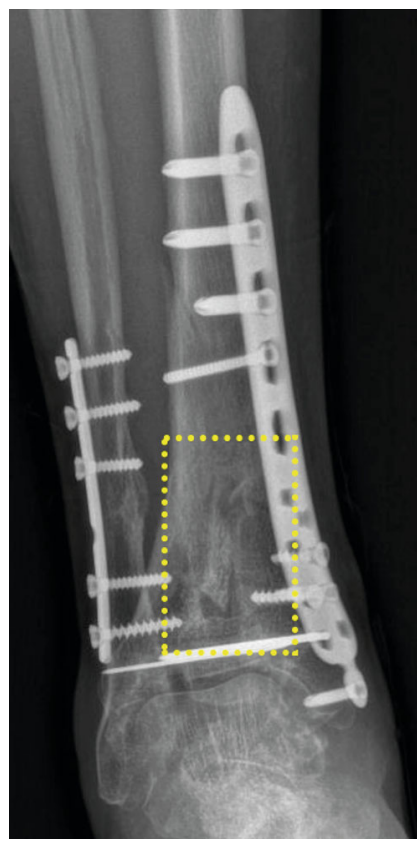

(c)

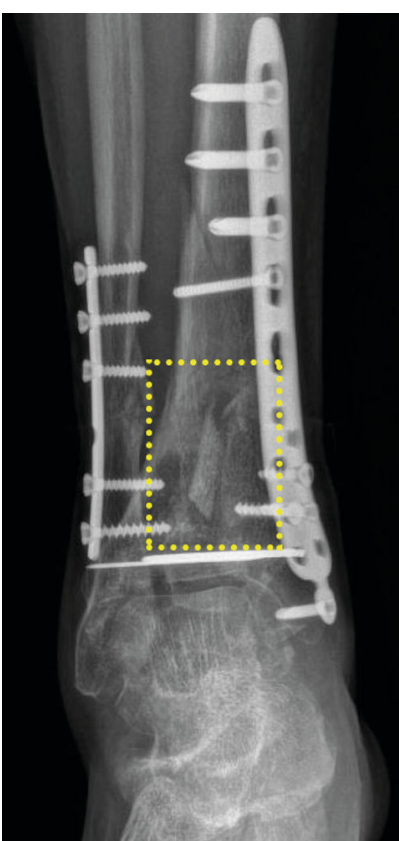

(b)

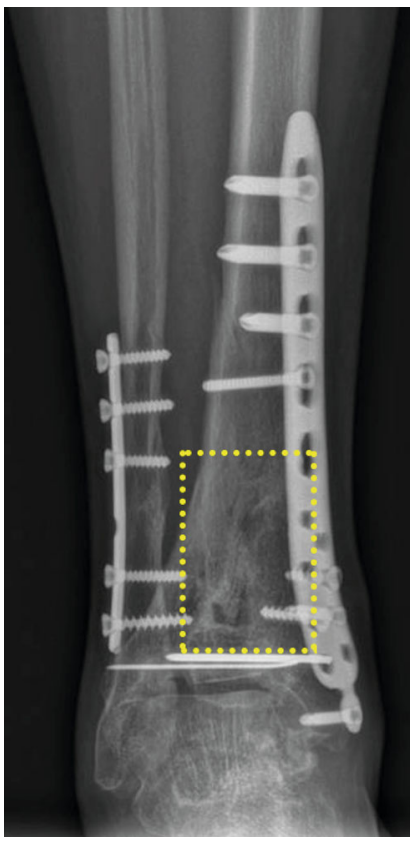

(d)

FIGURE 1: Plain radiographs of the right crus taken at the time when the patient was (a) admitted to the rehabilitation center, (b) before LIPUS therapy, (c) 1 week after the end of LIPUS therapy, and (d) 2 months after the end of LIPUS. The yellow dotted line box indicates the right distal tibial comminuted fracture region.

increase proteoglycan formation in chondrocytes [11, 12]. Therefore, LIPUS can be an effective intervention applied in the treatment of fresh fractures and nonunions, which is approved by the U.S. Food and Drug Administration (FDA).

However, whether LIPUS promotes the formation of heterotopic ossification (HO) is still uncertain. $\mathrm{HO}$ is defined as pathological osseous tissue in the periarticular soft tissues after neurologic injury, orthopedic interventions, muscular injury, and fracture. Unlike tissue calcification due to calcium deposition, the formation of $\mathrm{HO}$, a complex and dynamic developmental process, is regulated by the neuroendocrine system [13]. Wijdicks et al. demonstrated the effect of LIPUS on ectopic bone formation induced by rhBMP-2 [14]. Therefore, it is necessary to avoid $\mathrm{HO}$ formation during the fracture healing process with the use of LIPUS. If HO occurs, the orthopedist will properly adjust the rehabilitation treatment. However, the early and accurate diagnosis of $\mathrm{HO}$ is challenging. 
In clinic, orthopedists usually diagnose $\mathrm{HO}$ by palpation and plain radiography during the rehabilitation of patients with trauma. Additionally, ultrasonography has been applied to diagnose HO. Some studies indicated that ultrasonography distinguished mature $\mathrm{HO}$ from the surrounding soft tissue with high specificity and effectively detected the immature $\mathrm{HO}$ [15-18]. Ultrasonography is useful to visualize $\mathrm{HO}$ lesion with several advantages, including nonionizing radiation, bedside real-time monitoring, repeatable detection, relatively simple operation, and low cost. A previous study demonstrated that the visualization of $\mathrm{HO}$ using ultrasonography revealed the development of $\mathrm{HO}$ in the muscle tissues around the injured joints [19]. Ultrasonography has potentials to provide guidance for orthopedists to make individualized rehabilitation therapy.

Therefore, in this study, we present a case with the nonunion after comminuted fracture with an aim to investigate the positive effect of LIPUS therapy on nonunion and to monitor HO occurrence that may be stimulated by LIPUS. Both radiographic and ultrasonographic outcomes suggest that LIPUS facilitates fracture repair without HO formation. In comparison with plain radiography, ultrasonography is a useful bedside tool for monitoring $\mathrm{HO}$ occurrence during the process of LIPUS therapy.

\section{Materials and Methods}

2.1. Statement of Informed Consent. This study was approved by the Ethics Committee of the Guangdong Work Injury Rehabilitation Center, Guangzhou, China. The patient provided his written informed consent to participate in this study.

2.2. Patient and Initial Therapy. A 42-year-old Chinese male patient with the functional limitation and pain of the right lower limb was admitted to the rehabilitation center, approximately 1 month after his plate internal fixation operation for comminuted fracture of his left calcaneus, right tibia, and fibula performed at another hospital. The physical examination revealed a poor healing of surgical incision of $12 \mathrm{~cm}$ with eschar on the anterior side of the right tibia. No exudate was found but the skin surrounding the surgical incision was red and rigid. The range of motion of the left ankle, bilateral hip, and knee was normal, whereas the swollen right ankle had the limited range of motion (plantarflexion: $5^{\circ}$, dorsiflexion: $0^{\circ}$ ). The blood test showed the absence of abnormalities.

The patient was hospitalized. The plain radiography revealed a distinct fracture line in the right distal tibia, distal fibula surrounded with free bone fragments, and the fixations of fractures (Figure 1(a)). Then, kinesitherapy and occupational therapy were performed to improve his lower limb function and the range of ankle motion. Meanwhile, drug therapy, transcutaneous electrical nerve stimulation, and medium-frequency electrical stimulation were applied to promote blood circulation and to reduce inflammation and pain.

After approximately three and half months, the kirschner wire in the right medial malleolus was removed and the surgical debridement of the right crus was performed under

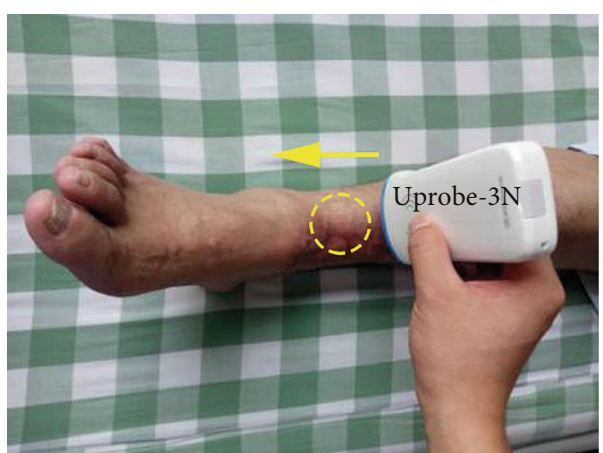

FIgURE 2: Ultrasound scanning of the right distal tibia using Uprobe-3N, a wireless ultrasound probe. The arrow indicates the scanning orientation. The dotted ellipse presents the site of LIPUS therapy.

local anesthesia. The aforementioned therapy measures continued. When the 5-month treatment was completed, the plain radiography was taken again to reveal the poor condition of fracture healing. Figure 1(b) clearly shows the defect still at the right distal tibial fracture area. The distal tibial fracture was then diagnosed as nonunion because it occurred 6 months after the fracture. Therefore, LIPUS therapy was suggested to perform LIPUS to accelerate the fracture healing.

2.3. LIPUS Therapy and Ultrasound Monitoring. An ultrasonic system (SXUltrasonic, Shenzhen, China) for accelerating fracture healing was specially designed for the LIPUS therapy in this study. The acoustic parameters including the output power, duty cycle, pulse width, and pulse repetition frequency (PRF) were adjustable. In this study, the ultrasound transducer with a central frequency of $1.5 \mathrm{MHz}$ generated ultrasound waves with a $200 \mu$ s pulse width, a $20 \%$ duty cycle, a $1 \mathrm{kHz} \mathrm{PRF}$, and a power of $30 \mathrm{~mW} / \mathrm{cm}^{2}$. The handheld transducer was placed on the site of the right anterior tibial fracture with enough coupling gel interposed between the transducer and the skin. The bedside LIPUS therapy performed by one investigator would last for 20 minutes and 5 times a week for 6 weeks. During the therapy, the patient should keep his right lower limb immobilized. Besides the LIPUS therapy, the aforementioned kinesitherapy and occupational treatments were performed as well.

Due to the fracture site neared entheses of extensor tendon and ankle joint, where $\mathrm{HO}$ might easily occur, it was necessary to monitor the LIPUS-treated bone healing process and to avoid $\mathrm{HO}$ formation. The ultrasound monitoring was performed once per week. A wireless ultrasound probe (Uprobe-3N, linear transducer, SonoStar, Guangzhou, China) with a fixed central frequency of $10 \mathrm{MHz}$ was placed perpendicular to the distal tibia and scanned along the long axis of tibia (Figure 2). The enough coupling gel was interposed between the transducer and the skin.

\section{Results of Diagnostic Assessment}

As shown in Figure 3, there was a distinct defect of the right distal tibia (Figure 3(a)) 2 weeks after LIPUS therapy, and the 


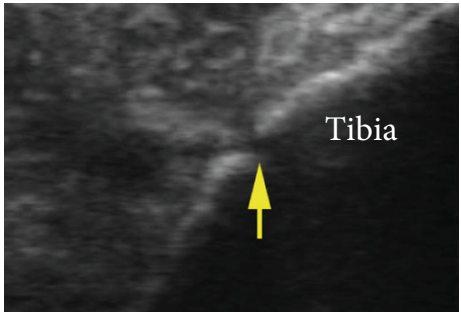

(a)

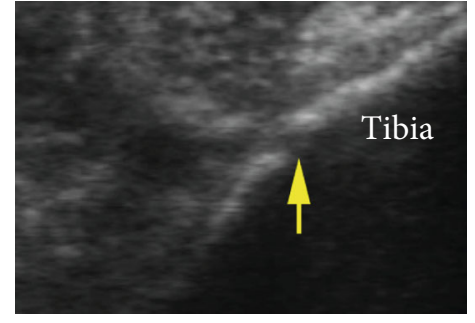

(b)

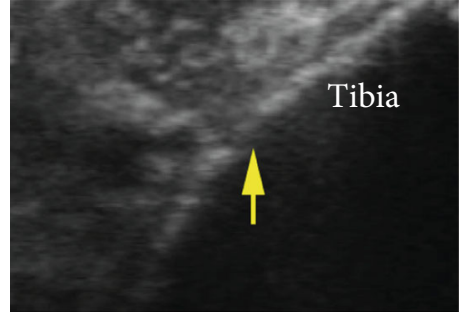

(c)

FIgURe 3: Ultrasound images at the right distal tibia examined, respectively, (a) 2 weeks, (b) 4 weeks, and (c) 6 weeks after the commence of LIPUS therapy. The arrow indicates the defect of the tibia.

gap became poorly demarcated with the therapy time increasing (at 4 weeks after LIPUS therapy) (Figure 3(b)). After the 6-week LIPUS therapy, the mineralized bone formation in the area of defect of the right distal tibia was observed in an ultrasound image (Figure 3(c)). Meanwhile, during the LIPUS therapy, ultrasound monitoring showed no evidence of calcification lesion within soft tissue.

The ultrasonographic outcomes were consistent with those in plain radiography (Figure 1). Figure 1(c) shows callus formation and the blurred fracture line in the same area exposed to LIPUS. Two weeks after the end of LIPUS therapy, the patient left the hospital to recuperate at home. At 2 months after the end of LIPUS therapy, the patient went to the rehabilitation center for reexamination and presented symptoms relief of pain, swelling, and motion limitation, but mild soreness of ankle joint after a long walk. The plain radiography showed the callus formation in most of the fracture area (Figure 1(d)), and traumatic arthritis was diagnosed at the ankle joint.

\section{Discussion}

In comparison with the surgical interventions in the treatment of nonunion, LIPUS tends to be a relatively safe, noninvasive, and inexpensive method with fewer potential complications. LIPUS may be suggested when the conservative treatment is required or the surgical operations are of high risk $[2,5]$. Gebauer et al. reported that the healing rate of nonunion treatment by LIPUS could reach up to $85 \%$ similar to that of the surgical methods [4]. The radiographic and ultrasonographic outcomes of this study revealed signs of progressive bone repair and suggested that LIPUS promoted fracture union.

Lower limbs support the human body and participate in the completion of walking, running, bouncing, and other movements. Therefore, the musculoskeletal system of lower limbs usually experiences mechanical force and is influenced by the mechanical environment. After long-term uselessness of the muscles and bones, atrophy, osteoporosis, and dysfunction tend to occur. Long-term immobilization of a patient with fracture contributes to the lack of mechanical force of the injury site; ultrasonic waves however produce mechanical force to improve the mechanical environment. The improvement of the mechanical environment may stimulate endochondral ossification on which one mechanism of fracture healing is based $[20,21]$. However, a previous study reported that HO similarly occurred based on endochondral ossification [13]. Therefore, LIPUS may induce the formation of $\mathrm{HO}$ by enhancing endochondral ossification. During the process of LIPUS therapy, it needs ultrasonography to monitor HO formation.

The patient involved in this study suffered distal tibial comminuted fracture with a worse prognosis (dysfunctions of right crus, swelling, inflammation, and pain). Although he underwent 5-month kinesitherapy and drug treatment, the fracture did not heal and thus needed an intervention. LIPUS therapy is noninvasive but with one potential complication of $\mathrm{HO}$ occurrence because of the fracture site near entheses of extensor tendon and ankle joint. Ultrasonography was applied to monitor the fracture healing and $\mathrm{HO}$ occurrence. If any indications present the potential of $\mathrm{HO}$ in soft tissues, LIPUS therapy will stop. Fortunately, LIPUS stimulated no $\mathrm{HO}$ in this study.

\section{Conclusions}

This study reports a feasibility study of fracture nonunion treated with LIPUS and monitored with ultrasonography. The results suggest that ultrasonography is a potential tool to guarantee the performance of LIPUS therapy with monitoring $\mathrm{HO}$ formation. Easy to use, the integration of the handheld ultrasound scanner and the ultrasonic therapeutic apparatus is entirely dedicated to help orthopedists make high-quality care and diagnosis.

\section{Data Availability}

All datasets generated for this study are included in the article.

\section{Conflicts of Interest}

All authors declare no conflicts of interest in this paper.

\section{Acknowledgments}

We acknowledge the support by the Project of Science and Technology Department of Guangdong Province (grant number 2016A020216017) and the National Natural Science Foundation of China (grant number 81371560). 


\section{References}

[1] J. A. Bishop, A. A. Palanca, M. J. Bellino, and D. W. Lowenberg, "Assessment of compromised fracture healing," The Journal of the American Academy of Orthopaedic Surgeons, vol. 20, no. 5, pp. 273-282, 2012.

[2] Y. H. Mirza, K. H. Teoh, D. Golding, J. F. Wong, and Y. Nathdwarawala, "Is there a role for low intensity pulsed ultrasound (LIPUS) in delayed or nonunion following arthrodesis in foot and ankle surgery?," Foot and Ankle Surgery, vol. 25, no. 6, pp. 842-848, 2019.

[3] P. R. Mandt and D. H. Gershuni, "Treatment of nonunion of fractures in the epiphyseal-metaphyseal region of long bones," Journal of Orthopaedic Trauma, vol. 1, no. 2, pp. 141-151, 1987.

[4] D. Gebauer, E. Mayr, E. Orthner, and J. P. Ryaby, "Low-intensity pulsed ultrasound: effects on nonunions," Ultrasound in Medicine \& Biology, vol. 31, no. 10, pp. 1391-1402, 2005.

[5] R. Leighton, J. T. Watson, P. Giannoudis, C. Papakostidis, A. Harrison, and R. G. Steen, "Healing of fracture nonunions treated with low-intensity pulsed ultrasound (LIPUS): a systematic review and meta-analysis," Injury, vol. 48, no. 7, pp. 1339-1347, 2017.

[6] M. J. Gardner, J. M. Evans, and R. P. Dunbar, "Failure of fracture plate fixation," The Journal of the American Academy of Orthopaedic Surgeons, vol. 17, no. 10, pp. 647-657, 2009.

[7] T. K. Kristiansen, J. P. Ryaby, J. Mccabe, J. J. Frey, and L. R. Roe, "Accelerated healing of distal radial fractures with the use of specific, low-intensity ultrasound. A multicenter, prospective, randomized, double-blind, placebo-controlled study," The Journal of Bone \& Joint Surgery, vol. 79, no. 7, pp. 961-973, 1997.

[8] A. A. Pilla, M. A. Mont, P. R. Nasser et al., "Non-invasive lowintensity pulsed ultrasound accelerates bone healing in the rabbit," Journal of Orthopaedic Trauma, vol. 4, no. 3, pp. 246-253, 1990.

[9] N. M. Rawool, B. B. Goldberg, F. Forsberg, A. A. Winder, and E. Hume, "Power Doppler assessment of vascular changes during fracture treatment with low-intensity ultrasound," Journal of Ultrasound in Medicine, vol. 22, no. 2, pp. 145-153, 2003.

[10] J. T. Ryaby, J. Mathew, and P. Duarte-Alves, "Low intensity pulsed ultrasound affects adenylate cyclase activity and TGF$\beta$ synthesis in osteoblastic cells," Transactions of the 38th Annual Meeting of the Orthopaedic Research Society, vol. 7, pp. 590-597, 1992.

[11] K. H. Yang, J. Parvizi, S. J. Wang et al., "Exposure to lowintensity ultrasound increases aggrecan gene expression in a rat femur fracture model," Journal of Orthopaedic Research, vol. 14, no. 5, pp. 802-809, 1996.

[12] J. Parvizi, C. C. Wu, D. G. Lewallen, J. F. Greenleaf, and M. E. Bolander, "Low-intensity ultrasound stimulates proteoglycan synthesis in rat chondrocytes by increasing aggrecan gene expression," Journal of Orthopaedic Research, vol. 17, no. 4, pp. 488-494, 1999.

[13] D. Dey, B. M. Wheatley, D. Cholok et al., "The traumatic bone: trauma-induced heterotopic ossification," Translational Research, vol. 186, pp. 95-111, 2017.

[14] C. A. Wijdicks, A. S. Virdi, K. Sena, D. R. Sumner, and R. M. Leven, "Ultrasound enhances recombinant human BMP-2 induced ectopic bone formation in a rat model," Ultrasound in Medicine \& Biology, vol. 35, no. 10, pp. 1629-1637, 2009.
[15] M. Kara, S. Yalçın, D. Yenigün, T. Tiftik, F. Ü. Malas, and L. Özçakar, "Heterotopic ossification and cubital tunnel syndrome in traumatic brain injury: ultrasound 'sees' both," Journal of Back and Musculoskeletal Rehabilitation, vol. 28, no. 2, pp. 415-417, 2015.

[16] S. S. Onat, Z. Özişle, A. Orhan, B. Akman, K. Köklü, and L. Özçakar, "Ultrasonographic diagnosis of heterotopic ossification and secondary nerve entrapments in a patient with spinal cord injury," Medical Ultrasonography, vol. 19, no. 3, pp. 338-339, 2017.

[17] P. Falsetti, C. Acciai, R. Palilla, F. Carpinteri, C. Patrizio, and L. Lenzi, "Bedside ultrasound in early diagnosis of neurogenic heterotopic ossification in patients with acquired brain injury," Clinical Neurology and Neurosurgery, vol. 113, no. 1, pp. 2227, 2011.

[18] T. Rosteius, E. M. Suero, D. Grasmücke et al., "The sensitivity of ultrasound screening examination in detecting heterotopic ossification following spinal cord injury," Spinal Cord, vol. 55, no. 1, pp. 71-73, 2017.

[19] Q. Wang, P. Zhang, P. Li et al., "Ultrasonography monitoring of trauma-induced heterotopic ossification: guidance for rehabilitation procedures," Frontiers in Neurology, vol. 9, p. 771, 2018.

[20] Z. Thompson, T. Miclau, D. Hu, and J. A. Helms, "A model for intramembranous ossification during fracture healing," Journal of Orthopaedic Research, vol. 20, no. 5, pp. 1091-1098, 2002.

[21] P. A. Nolte, J. Klein-Nulend, G. H. R. Albers et al., "Low-intensity ultrasound stimulates endochondral ossification in vitro," Journal of Orthopaedic Research, vol. 19, no. 2, pp. 301-307, 2001. 